\title{
BMJ Open Evidence on health-promoting lifestyle practices and information and communication technologies: scoping review protocol
}

\author{
Elizabeth M Joseph-Shehu, ${ }^{1,2}$ Busisiwe P Ncama ${ }^{1}$
}

To cite: Joseph-Shehu EM, Ncama BP. Evidence on health-promoting lifestyle practices and information and communication technologies: scoping review protocol. BMJ Open 2017;7:e014358. doi:10.1136/bmjopen-2016014358

- Prepublication history for this paper is available online. To view these files please visit the journal online (http://dx.doi.org/10.1136/ bmjopen-2016-014358).

Received 20 September 2016 Revised 10 December 2016 Accepted 25 January 2017

\section{CrossMark}

\section{${ }^{1}$ School of Nursing and Public Health, University of KwaZulu-Natal, Howard College, Durban, South Africa ${ }^{2}$ Faculty of Health Sciences, National Open University of Nigeria, Jabi, Abuja, Nigeria}

\section{Correspondence to}

Elizabeth M Joseph-Shehu; ejoseph-shehu@noun.edu.ng; lizjoe26@gmail.com

\section{ABSTRACT}

Introduction: Information and communication technologies (ICTs) play a key role in improving health and maintaining health promoting behaviours. ICTs are therefore one potential solution for promoting healthy lifestyles. In addition, they can assist in the reduction and control of the menace of both communicable and non-communicable diseases. This study will map evidence of interventions that demonstrate the effect of ICTs on health-promoting lifestyle practices that can prevent and control diseases. It is anticipated that this study will help identify areas where there is need for primary research.

Methods and analysis: The following electronic databases will be searched: PsycArticle (EBSCO), PsycINFO (EBSCO), Science direct, PubMed, Medline (EBSCO) and Google Scholar. The study will be conducted in two stages: the first stage will map out the studies descriptively while the second stage will map the additional inclusion criteria of quality assessment. Two independent reviewers will undertake the data extraction. Relevant outcomes of the studies will be analysed thematically using NVIvo computer software. The authors will code all evidence independently. Thereafter the authors will critically cross-examine the relationship of the research questions to the emerging themes from the selected articles. The authors hope to find a large number of studies on health-promoting lifestyles that encompass six-subscales of health-promoting activities (nutrition, stress management, interpersonal relation, selfactualisation, health responsibility, physical activity) and ICT.

Dissemination: This study will be presented in conferences related to health promotion and health-promoting lifestyles. It will also be disseminated in print and electronically.

Trial registration number: CRD42016042568.

\section{INTRODUCTION}

Globally, health promotion is used as an umbrella concept for both prevention of disease and promotion of health. ${ }^{1}$ It is a
Strengths and limitations of this study

- There are limited studies that link healthpromoting lifestyle practices (HPLPS) to information and communication technologies.

- There are gaps in studies that address all the subscales of the health-promoting lifestyle profile.

- The study will be restricted to literature published only in the English language.

- Only literature published from January 2007 to July 2016 will be included in the study.

- The outcomes of the study will be effective in that they will sustain HPLPS.

means of enabling people to have control over the determinants of health and hence achieve maximum health. ${ }^{2}$ Health promotion views health as a product of daily life and explicitly lists some prerequisites for health. ${ }^{3}$ Zhang $e t a t^{4}$ emphasised the need to improve lifestyle in maintaining a healthy life. Health-promoting lifestyle behaviours focus on enhancing, sustaining and increasing an individual's level of well-being, selfactualisation and personal fulfilment ${ }^{5} \quad 6$ which brings about optimal health.

Information and communication technologies (ICTs) have helped to improved health promotion interventions. According to While and Dewsbury, ${ }^{7}$ the US National Broadband Plan identified a key role for ICTs in improving health and healthcare through enhancing care delivery, coordination and engagement with patients. The use of ICTs in disease treatment, preventive vaccination, medical appointments, and medication for long-term self-management has had a positive influence on healthcare delivery. ${ }^{8}$ Despite the benefits of ICTs in all spheres of life, there are adverse effects associated with the use of ICTs if there are no checks and balances on the implementation of the 
programmes. For example, the increase in the development of technology has made many academic staff adopt sedentary lifestyles which make them physically inactive. ${ }^{9}{ }^{10}$ Inactivity has been linked to most of the non-communicable diseases (NCDs) that threaten public health globally. ${ }^{11}$ NCDs account for $80 \%$ of the adult disease burden in developed countries and $70 \%$ in middle-income countries, while almost $50 \%$ of the adult disease burden is attributed to NCDs in high mortality regions in the world. ${ }^{12}$ Boutayeb and Boutayeb ${ }^{12}$ have further documented that by 2020 NCDs will be causing seven out of every 10 deaths in lower and middle income countries. These only relate to physical inactivity; in addition, there is evidence that other components of the health-promoting lifestyle profile (nutrition, stress management, interpersonal relation, self-actualisation, health responsibility, physical activity) also contribute greatly to health promotion and disease prevention. Bully et $a l^{13}$ documented that recent studies have attributed health-promoting lifestyle practices (HPLPs) and moderate alcohol intake to reductions in mortality rates related to NCDs by $50 \%$ and increases in life expectancy by more than 11 years.

Despite the availability of literature and campaigns on the benefits of health-promoting behaviour, there are still gaps relating to HPLPs that can increase the life expectancy and quality of life of individuals. The healthcare systems pay little attention to lifestyle factors. The focus of healthcare systems is mostly on curative rather than on preventive health. All federal institutions in Nigeria are now beneficiaries of the National Health Insurance Scheme, but most of the care rendered by this scheme is curative rather than preventive.

Little is known about the use of ICTs for comprehensive HPLPs that can contribute greatly to the prevention and control of non-communicable and also some communicable diseases among adults in low and middle income countries such as Nigeria. Hence, there is a need to map available evidence through a scoping review.

\section{RESEARCH OBJECTIVES}

The main aim of the review is to establish and map out evidence of interventions that relate to ICTs and effective and efficient HPLPs that can prevent and control diseases as well as to identify areas required to conduct primary research. The specific objectives of the study include the following:

- To review evidence that links the use of ICT in HPLPs that improve the quality of life and increase life expectancy

- To review evidence where HPLPs in combination with ICT improve health status (physical and mental health)

- To review evidence relating to the incorporation of ICT into health-promoting lifestyle interventions that can sustain HPLPs.
The study will provide answers to the following questions:

- What evidence exists that links the use of ICT to HPLPs to improve the quality of life and to increase life expectancy?

- What evidence exists that HPLPs in combination with ICT improve physical and mental health?

- Is there evidence that incorporating ICT into healthpromoting lifestyle intervention will sustain behavioural modification that will last?

\section{METHODS/DESIGNS}

This study protocol was registered and published with the International Prospective Register of Systematic Reviews (PROSPERO) (registration number CRD42016042568).

\section{SEARCH STRATEGY AND STUDY SELECTION}

The following electronic databases will be searched for the study: PsycArticle (EBSCO), PsycINFO (EBSCO), Science direct, PubMed, Medline (EBSCO) and Google Scholar. The search strategy will include all studies that addressed the intervention irrespective of the type of studies and study designs. All peer-reviewed studies and grey literature that addresses the research questions will be selected in the study. Only articles published in English between 2007 to date will be used. The literature search results will be uploaded to EndNote X7. The EndNote X7 software will be used to find and remove duplicates.

\section{CRITERIA FOR INCLUSION}

- Articles published in the English language

- Literature published from January 2007 to July 2016

- Studies with focus on well adults, workers, professionals

- Evidence from published relevant interventions

- Studies reporting on intervention(s) such as any of the subscales of the health-promoting profile (nutrition, physical activity, stress management, interpersonal relationships, self-actualisation and health responsibility) and ICT

- Review articles including: systematic reviews, metaanalysis, scoping reviews, peer-reviewed journal articles and rapid reviews

- Grey literature sources such as: documents from government and non-governmental organisations and academic dissertations

- All types of study designs such as cohort studies, crosssectional studies, qualitative studies, quantitative studies, randomised control trial studies, quasi-experimental study designs and pilot studies.

\section{EXCLUSION CRITERIA}

- Intervention that does not include any form of ICT

- Studies focusing on diseases, youth, students or children 
Table 1 Electronic search records

Date Keyword Search engine Number of publications searched used retrieved

- Studies not including all or any of the subscales of the health-promoting lifestyle profile

- Studies reporting on either alcoholism or cigarette smoking

- Non-English publications

- Articles published before January 2007 and after July 2016

- Articles not focusing on adults.

Keywords search (table 1) will be combined into a phrase including Boolean (AND, OR) terms, as follows: (health promoting lifestyle profile OR health promoting lifestyle behaviour OR wellness OR nutrition OR physical activity OR interpersonal relationships OR health responsibility OR stress management OR selfactualisation, and information and communication technology OR ICT OR mobile phone OR text messages OR SMS OR the internet).

Updated records of the number of publications identified during each session of the literature search and date will be kept by all the members of the research team using the information in table 1.

\section{DATA EXTRACTION}

- Numerical summary and thematic analysis will be employed to extract background information from the selected studies. In order to answer the research questions as guided by Population, Interventions, Comparison and Outcome (PICO) (table 2), the reviewers will collectively design a data-chronicling form to determine the text words, and themes to include and extract. They will also collectively develop the data-charting form.

- Information and data relevant to answer the research questions will be determined by the reviewers collectively.
- Update recording of data relevant to the study.

- Relevant data will be extracted from all the eligible studies by two independent reviewers in duplicate. Data to be extracted include: (i) author (s) names; (ii) year of publication; (iii) study design and/or methodology; (iv) study population; (v) intervention (s); (vi) study setting; (vii) aim of the study; (viii) geographic location of the study; (ix) primary and secondary outcome of the study; (x) conclusions. Information specific to HPLPs and description of the intervention (ICT) will also be extracted. If necessary, a third reviewer will be consulted in order to reach consensus.

- The researchers will collectively carry out a thematic analysis to extract relevant outcomes using NVivo software.

\section{ANALYSIS AND RESULTS}

Narrative synthesis of the outcomes of the selected articles will be presented in the study. The researchers will employ the following steps in analysing and reporting findings from the study.

I. Author (s) names, year of publication, study design and/or methodology, study population, intervention (s), study setting, aim of the study, geographic location of the study and outcome of the study will be examined.

II. Findings from the studies on HPLPs of adults, evidence on effective and efficient HPLP intervention using ICT; evidence on effective and efficient HPLP intervention using ICT will be compared with evidence on effective and efficient HPLC intervention without ICT. The researchers will code all the findings independently. Thereafter the researchers will collectively assess the themes and conduct a critical appraisal of their relationship to the research questions. Furthermore, the authors will also examine the meaning of the findings in relation to the aim of the study as well as its implications for research, practice and policy.

Table 2 Population, Interventions, Comparison, Outcome and Study setting (PICOS) framework for determination of the eligibility of the review questions

\begin{tabular}{ll}
\hline Criteria & Determinants \\
\hline Population & $\begin{array}{l}\text { The population for this study will be healthy adults, workers, well individuals. Patients, children, } \\
\text { students, adolescents (youth will be excluded from this study) }\end{array}$ \\
Interventions & $\begin{array}{l}\text { Health-promoting lifestyle profile (nutrition, physical activity, interpersonal relationship, health } \\
\text { responsibility, stress management, self-actualisation) and information and communication technologies } \\
\text { (ICT, mobile phone, text messages, SMS, internet, etc) }\end{array}$ \\
Comparison & Health-promoting lifestyle profile intervention without ICT \\
Outcomes & Effective and sustaining health-promoting lifestyle practices and health status (normal weight, normal \\
blood pressure, normal blood sugar and good mental and physical health)
\end{tabular}




\section{DISCUSSION}

This study will generate evidence that will help to describe the link between health-promoting lifestyle profiles and ICT in achieving a behavioural modification that will endure and enable the individual to have a quality of life that will, in turn, increase his or her life expectancy.

Health-promoting lifestyle profiles and ICTs have received little research attention. Findings from studies on just two subscales, nutrition and physical activity, have shown that if individuals maintain a healthy nutritional diet and participate in low to moderate physical activity their quality of life is improved. Therefore, one can argue that having a good healthy lifestyle that encompasses all of the six subclasses of the health-promoting lifestyle profile will have much more impact. However, measures to increase our understanding of the roles of these other subclasses of health-promoting lifestyle profiles, and their relationship to improving the quality of life and increasing life expectancy, have not been given much attention. This study will help researchers to address these issues and also describe the relationship between the phenomena comprehensively. Effective and efficient health-promoting lifestyles that prevent and control diseases (both non-communicable and communicable) is an area that needs more attention. We are not aware if there are any empirical studies or reviews that have been done in low and middle income countries that answer the specific questions outlined in the current proposal.

Therefore, findings from this study will contribute to the body of knowledge on health-promoting lifestyle profiles and ICTs which will have a positive impact on research, practice and policy in the area of health promotion that should improve the quality of life of individuals and increase their life expectancy. Due to the paucity of data in the literature regarding this topic, we intend to include all studies from anywhere in the world that address our types of interventions and population. Our argument is that if an individual has been practising a health-promoting lifestyle effectively and efficiently, such an individual will not have issues with medication or dietary compliance.

Findings from this scoping review will play a vital role in the primary, secondary and tertiary prevention of diseases. In addition, evidence generated from this study will provide the building blocks for effective and efficient HPLPs that will help in the primary prevention of disease, and also add to the existing literature in health promotion and ICT. Lastly it will also outline aspects of the study that require meta-analysis and primary empirical study.

Contributors EMJ-S: conception (generator of the review) and design; writing the manuscript. BPN: critical revision of the manuscript and administrative and technical support.

Competing interests None declared.

Provenance and peer review Not commissioned; externally peer reviewed.

Data sharing statement The manuscript is a protocol for a scoping review and there are no data currently. We would be happy to share the dataset after the completion of the study.

Open Access This is an Open Access article distributed in accordance with the Creative Commons Attribution Non Commercial (CC BY-NC 4.0) license, which permits others to distribute, remix, adapt, build upon this work noncommercially, and license their derivative works on different terms, provided the original work is properly cited and the use is non-commercial. See: http:// creativecommons.org/licenses/by-nc/4.0/

\section{REFERENCES}

1. Nilsson $\mathrm{P}$, Andersson $\mathrm{IH}$, Ejlertsson $\mathrm{G}$, et al. Workplace health resources based on sense of coherence theory. Int $J$ Workplace Health Manag 2012;5:156-67.

2. Torp S, Vinje HF. Is workplace health promotion research in the Nordic countries really on the right track? Scand J Public Health 2014;42(15 Suppl):74-81.

3. Potvin L, Jones CM. Twenty-five years after the Ottawa Charter: the critical role of health promotion for public health. Can J Public Health 2011:102;244-8.

4. Zhang SC, Wei CN, Fukumoto $\mathrm{K}$, et al. A comparative study of health-promoting lifestyles in agricultural and non-agricultural workers in Japan. Environ Health Prev Med 2011;16:80-9.

5. Tsai YC, Liu CH. Factors and symptoms associated with work stress and health-promoting lifestyles among hospital staff: a pilot study in Taiwan. BMC Health Serv Res 2012;12:199.

6. Nassar OS, Shaheen AM. Health-promoting behaviours of university nursing students in Jordan. Health 2014;6:2756.

7. While A, Dewsbury G. Nursing and information and communication technology (ICT): a discussion of trends and future directions. Int J Nurs Stud 2011;48:1302-10.

8. Cocosila M, Archer N. Adoption of mobile ICT for health promotion: an empirical investigation. Electronic Markets 2010;20:241-50.

9. Pirincci E, Rahman S, Durmuş A, et al. Factors affecting health-promoting behaviours in academic staff. Public health 2008;122:1261-3.

10. Turkmen M, Ozkan A, Murat K, et al. Investigation of the relationship between physical activity level and healthy life-style behaviors of academic staff. Educ Res Rev 2015;10:577-81.

11. de Rezende LF, Rabacow FM, Viscondi JY, et al. Effect of physical inactivity on major noncommunicable diseases and life expectancy in Brazil. J Phys Act Health 2015;12:299-306.

12. Boutayeb A, Boutayeb S. The burden of non communicable diseases in developing countries. Int $J$ Equity Health 2005;4:2.

13. Bully P, Sánchez Á, Zabaleta-del-Olmo E, et al. Evidence from interventions based on theoretical models for lifestyle modification (physical activity, diet, alcohol and tobacco use) in primary care settings: a systematic review. Prev Med 2015;76 (Suppl):S76-93. 